\title{
Assessment of Soil Physical Quality Indicators in Rice Soils of Cuddalore District of Tamil Nadu, India
}

\author{
L. Seevagan $^{1 *}$, R.K. Kaleeswari ${ }^{1}$, M.R. Backiyavathy ${ }^{1}$ and D. Balachandar ${ }^{2}$ \\ ${ }^{1}$ Department of Soil Science and Agricultural Chemistry, Tamil Nadu Agricultural University, \\ Coimbatore - 641 003, India \\ ${ }^{2}$ Department of Agricultural Micro Biology, TNAU, Coimbatore - 3, India \\ *Corresponding author
}

\section{A B S T R A C T}

Keywords

Physical quality indicator, Principal component analysis, Rice soil, Soil quality, Physicochemical property, Biological property

Article Info

Accepted:

14 June 2020

Available Online:

10 July 2020
An investigation was conducted in intensive rice growing block of Viruthachalam, Cuddalore district of Tamil Nadu. The major objectives were to assess the soil physico- chemical and biological quality parameters in rice soils and to compare soil quality indexing methods viz., Principal component analysis, Minimum data set and Indicator scoring method and to develop soil quality indices for formulating soil and crop management strategies. To fulfil these objectives a total of 34 soil samples were collected from Viruthachalam block and TNAU research stations of Cuddalore district. The results obtained from PCA indicated five Principal Components ( PCs) with eigen values greater than 1 and soil variables from each PC were considered for minimum soil data set MDS) The soil parameters selected from PC 1, PC2, PC3, PC4,PC5 were, bulk density, particle density, porosity, WHC, sand, aggregate stability and Mean Weight Diameter.

\section{Introduction}

Globally, the area of rice (Oryza sativa L.) production has increased from 148 Mha in 2002 to 164 Mha in 2011(FAOSTAT2013). Asia is the main continent where this expansion has been reported. Food and nutritional security in Asian countries depend largely upon rice, because it is the source of $15 \%$ of protein and $21 \%$ of energy intake for the population (Depa et al., 2011). However, productivity of rice in lowland cultivated areas is low because of declining soil fertility (Haefele et al., 2014), degradation of soil structure (Das et al., 2014a) and unreliable water resources, lack of resources and wide spread poverty (Das et al., 2014b)

Assessing the quality of soil resources has been stimulated by increasing awareness that 
it is an important component of the earth's biosphere, functioning not only in the production of food and fiber but also in ecosystems services and the maintenance of local, regional, and global ecological balance (Glanz, 1995). Soil quality primarily describes the combination of physical, chemical and biological characteristics that enables soils to perform a wide range of ecological functions (Karlen et al., 1997). The functions largely include, sustaining biological activity and diversity; regulating and partitioning water and solute flow; filtering, buffering, degrading, immobilizing and detoxifying organic and inorganic toxic materials; storing and cycling nutrients in soil-plant-atmospheric continuum and providing support of socio-economic treasures. Another way we can tell the quality of a soil is an assessment of how it performs all of its functions now and how those functions are being persuaded in future.

This capacity of the soil to function can be assessed by physical, chemical and/or biological properties, which in this context are known as soil quality indicators (Wander and Bollero, 1999). Perceptions of what constitutes a good soil vary. They depend on individual priorities with respect to soil function, intended land use and interest of the observer (Doran and Parkin, 1994, Shukla et al., 2006). Soil quality changes with time can indicate whether the soil condition is sustainable or not (Arshad and Martin, 2002, Doran, 2002).

Maintaining soil quality at a desirable level is a very complex issue due to climatic, soil, plant, and human factors and their interactions and it is especially challenging in lowland rice cropping systems because of puddling practices in soil preparation (Chaudhury et al., 2005). Hence the present study was conducted to assess the the soil quality indicators of rice soils.

\section{Materials and Methods}

\section{Study area}

The areas under intensive rice cultivation (>1.0 lakh ha ) in Tamil Nadu were selected for the study. In Tamil Nadu intensive rice producing districts were identified. Two sampling grids (10x10 sq.km) were used, with sampling depth of $10-15 \mathrm{~cm}$ soil sampling was carried out in locations which were subjected to various management strategies. The composite soil samples were analyzed for soil quality parameters.

The study was conducted in Virudhachalam block of Cuddalore district, Tamil Nadu and TNAU research station in this district.

The general geological formation of the district is simple with metamorphic rocks belonging to the gneiss family. Resting on these are the three great groups of sedimentary rocks belonging to different geological periods and overlaying each other in regular succession from the coast on the east to the hills on the west.

The area receives total rainfall of $1104 \mathrm{~mm}$. It includes both the south west $(373 \mathrm{~mm})$ and north east $(731 \mathrm{~mm})$ monsoons. The maximum recorded temperature of the district is $36.8^{\circ} \mathrm{C}$ while minimum temperature is $19.9^{\circ} \mathrm{C}$

The soils of the district can be divided into three main classes namely, the black soil, the red ferruginous and the arenaceous.

The black soil prevails largely in the Chidambaram, Vriddhachalam and Cuddalore Taluks. The arenaceous occurs chiefly near the coast in the Chidambaram and Cuddalore. Black clay is the most fertile kind of soil, the loam is the next best and the red sand and arenaceous soils are the poorest. 
The major crops cultivated in Cuddalore district are paddy, sugarcane, maize, black gram, green gram and groundnut.

Physical quality indicators include bulk density, Particle Density (Core sampler method Gupta and Dakshinamurthi (1985), soil texture (International pipette method), Water holding Capacity (Piper 1966), soil aggregate stability and Mean weight diameter was determined by Yoder"s Modified weight sieving method (Yoder, 1936)

\section{Statistical analysis}

All the Statistical Analysis described in this chapter was performed using the softwares STATISTICA 10.0 and SPSS 20.0.

\section{Results and Discussion}

\section{Soil physical quality indicators}

Bulk density is an indicator of soil compaction and soil health. It affects infiltration rooting depth/restrictions, available water holding capacity and soil porosity. In Viruthachalam block of Cuddalore district, soil bulk density ranged from $1.10 \mathrm{Mg} \mathrm{m}^{-3}$ to $1.87 \mathrm{Mg} \mathrm{m}^{-3}$ To assess the effect of nutrient management strategies, soil quality parameters were assessed in rice soils of KVK, Viruthachalam. Lowest bulk density of 1.21 was registered due to the management practice of Integrated Nutrient Management (INM). This is an accordance with Mahajan et al., 2007 who reported that the low bulk density of surface soil was associated with relatively high organic matter content. Benefit of reduction in bulk density of the soil through the incorporation of organic matter has been well documented by Vasanthi and Kumarswamy (1999).

Particle density of rice soils of Viruthachalam block varied from $1.52 \mathrm{Mg} \mathrm{m}^{-3}$ to $3.85 \mathrm{Mg} \mathrm{m}^{-}$ ${ }^{3}$. Under management practice, SRI method registered the lowest particle density of 2.76 $\mathrm{Mg} \mathrm{m}^{-3}$ (Table 1).

Sand contributed the bulk of mechanical fractions of soil, which could be attributed to the dominance of sandy parent material. In Viruthachalam block of Cuddalore district, sand content varied from 12.00 per cent to 91.00 per cent. Under conventional rice farming, the lowest sand content of 24.00 per cent was registered. This result corroborate the findings of Balamurugan (2000).

Silt content of rice soils of Viruthachalam block ranged from 1.00 per cent to 78.00 . Highest silt content of 27.00 per cent was registered under conventional farming.

Variation in clay content reflected the corresponding differences in water retentivity, porosity and CEC requiring different management practices. In the present investigation, clay content varied from 6.00 percent to 61.00 per cent in Viruthachalam block. Among the crop management strategies, under organic cultivation of rice, highest clay content of 57.00 per cent was registered.

In the present study soil texture under different sites varied from clay loam to loam; however clay loam was the most dominant texture in Cuddalore district especially under paddy farming. Large variations in the soil texture might be due to the difference in nature and composition of parent material. A similar findings reported by Chander et al., (2014) and (Nayar et al., 2002).

Mean Weight Diameter (MWD) of rice soils of Viruthachalam block varied from 0.34 to 1.73 per cent (Table 2). Integrated Nutrient Management (INM) registered the highest MWD of 1.85 per cent. This result is in line with the findings of Sharma and Qaher (1989). 
Table.1 Physical indicators of Cuddalore district: Soil Bulk density $\left(\mathrm{Mg} \mathrm{m}^{-3}\right)$, Particle density $\left(\mathrm{Mg} \mathrm{m}^{-3}\right)$, Particle size distribution (per cent) and Soil texture

\begin{tabular}{|c|c|c|c|c|c|c|c|}
\hline Site.No & Name of the location & $\begin{array}{c}\text { Bulk } \\
\text { density }\end{array}$ & $\begin{array}{l}\text { Particle } \\
\text { density }\end{array}$ & $\begin{array}{c}\text { Sand } \\
(\%)\end{array}$ & Silt & $\begin{array}{c}\text { Sand } \\
(\%)\end{array}$ & Silt \\
\hline 1 & Viruthachalam & 1.40 & 1.64 & 33.00 & 14.00 & 52.00 & $\mathrm{cl}$ \\
\hline 2 & Kuppanatham & 1.50 & 1.52 & 28.00 & 15.00 & 56.00 & $\mathrm{cl}$ \\
\hline 3 & Kovilanur & 1.90 & 1.53 & 24.00 & 16.00 & 59.00 & $\mathrm{cl}$ \\
\hline 4 & M.Patti & 1.70 & 1.80 & 20.00 & 18.00 & 61.00 & $\mathrm{c}$ \\
\hline 5 & Manavalanallur & 1.82 & 1.92 & 45.00 & 13.00 & 41.00 & $\mathrm{cl}$ \\
\hline 6 & Mathur & 1.51 & 2.31 & 38.00 & 17.00 & 43.00 & $\mathrm{cl}$ \\
\hline 7 & Narumanam & 1.27 & 1.83 & 38.00 & 16.00 & 45.00 & $\mathrm{cl}$ \\
\hline 8 & Chinnaparur & 1.57 & 1.84 & 90.00 & 3.00 & 6.00 & sl \\
\hline 9 & Eadaiyur & 1.85 & 2.72 & 91.00 & 1.00 & 7.00 & $\mathrm{~s}$ \\
\hline 10 & Gopurapuram & 1.99 & 2.59 & 91.00 & 2.00 & 8.00 & $\mathrm{~s}$ \\
\hline 11 & Karnatham & 1.63 & 2.73 & 31.00 & 30.00 & 34.00 & $\mathrm{cl}$ \\
\hline 12 & Chinnakandiyankuppan & 1.09 & 2.59 & 32.00 & 33.00 & 31.00 & $\mathrm{cl}$ \\
\hline 13 & Ka.Elamangalam & 1.05 & 2.83 & 39.00 & 29.00 & 20.00 & $\mathrm{cl}$ \\
\hline 14 & Kattiyanallur & 1.84 & 2.64 & 38.00 & 29.00 & 21.00 & $\mathrm{cl}$ \\
\hline 15 & Earumanur & 1.66 & 2.32 & 41.00 & 33.00 & 22.00 & $\mathrm{cl}$ \\
\hline 16 & Kovilanur & 1.78 & 3.45 & 43.00 & 34.00 & 21.00 & $\mathrm{cl}$ \\
\hline 17 & Komangalam & 1.38 & 3.85 & 47.00 & 32.00 & 17.00 & $\mathrm{scl}$ \\
\hline 18 & Paravalur & 1.03 & 2.64 & 47.00 & 34.00 & 16.00 & $\mathrm{cl}$ \\
\hline 19 & Puliyur & 1.05 & 2.57 & 28.00 & 45.00 & 24.00 & $\mathrm{cl}$ \\
\hline 20 & Rajendirapatinam & 1.08 & 2.31 & 39.00 & 36.00 & 23.00 & $\mathrm{scl}$ \\
\hline 21 & Sathiyavadi & 1.09 & 2.25 & 44.00 & 38.00 & 14.00 & $\mathrm{scl}$ \\
\hline 22 & Puthukooraipettai & 1.67 & 2.90 & 24.00 & 45.00 & 26.00 & $\mathrm{cl}$ \\
\hline 23 & Peralaiyur & 1.10 & 1.79 & 30.00 & 46.00 & 21.00 & sl \\
\hline 24 & Mu.Agaram & 1.20 & 2.90 & 33.00 & 43.00 & 22.00 & $\mathrm{scl}$ \\
\hline 25 & Vetakudi & 1.30 & 2.58 & 40.00 & 44.00 & 14.00 & $\mathrm{scl}$ \\
\hline 26 & Thottikuppam & 1.17 & 1.56 & 12.00 & 78.00 & 14.00 & sl \\
\hline 27 & Siruvambar & 1.31 & 1.67 & 22.00 & 56.00 & 22.00 & $\mathrm{scl}$ \\
\hline 28 & Thoravalur & 1.86 & 2.26 & 34.00 & 52.00 & 14.00 & $\mathrm{sc}$ \\
\hline 29 & Sembalakurichi & 1.76 & 2.73 & 18.00 & 59.00 & 24.00 & $\mathrm{cl}$ \\
\hline 30 & Puthukooraipetai & 1.77 & 2.76 & 26.00 & 51.00 & 25.00 & $\mathrm{cl}$ \\
\hline \multirow[t]{4}{*}{31} & Rupanarayananallur & 1.63 & 2.98 & 47.00 & 14.00 & 14.00 & $\mathrm{cl}$ \\
\hline & Range & $\begin{array}{c}1.03- \\
1.99\end{array}$ & $\begin{array}{l}1.52- \\
3.85\end{array}$ & $\begin{array}{l}12.00- \\
91.00\end{array}$ & $\begin{array}{l}1.00- \\
78.00\end{array}$ & $\begin{array}{l}6.00- \\
61.00\end{array}$ & $\begin{array}{l}\text { clay } \\
\text { loam }\end{array}$ \\
\hline & Mean & 1.45 & 2.36 & 40.00 & 31.00 & 26.00 & \\
\hline & Standerd Deviation & 0.31 & 0.58 & 20.00 & 17.00 & 15.00 & \\
\hline
\end{tabular}


Table.2 Mean weight diameter (per cent) and available water holding capacity (per cent) of Cuddalore district

\begin{tabular}{|c|c|c|c|}
\hline Site.No & Name of the location & $\begin{array}{l}\text { Mean Weight Dia } \\
\text { meter \% }\end{array}$ & WHC\% \\
\hline 1 & Viruthachalam & 0.38 & 50.00 \\
\hline 2 & Kuppanatham & 0.41 & 52.00 \\
\hline 3 & Kovilanur & 0.34 & 55.00 \\
\hline 4 & M.Patti & 0.44 & 48.00 \\
\hline 5 & Manavalanallur & 0.47 & 40.00 \\
\hline 6 & Mathur & 0.48 & 47.00 \\
\hline 7 & Narumanam & 0.52 & 38.00 \\
\hline 8 & Chinnaparur & 0.54 & 46.00 \\
\hline 9 & Eadaiyur & 0.58 & 41.00 \\
\hline 10 & Gopurapuram & 0.56 & 43.00 \\
\hline 11 & Karnatham & 0.59 & 42.00 \\
\hline 12 & Chinnakandiyankuppan & 0.57 & 39.00 \\
\hline 13 & Ka.Elamangalam & 0.59 & 36.00 \\
\hline 14 & Kattiyanallur & 0.65 & 34.00 \\
\hline 15 & Earumanur & 0.72 & 45.00 \\
\hline 16 & Kovilanur & 0.55 & 52.00 \\
\hline 17 & Komangalam & 0.76 & 55.00 \\
\hline 18 & Paravalur & 0.78 & 37.00 \\
\hline 19 & Puliyur & 0.75 & 33.00 \\
\hline 20 & Rajendirapatinam & 0.83 & 44.00 \\
\hline 21 & Sathiyavadi & 0.86 & 42.00 \\
\hline 22 & Puthukooraipettai & 0.82 & 25.00 \\
\hline 23 & Peralaiyur & 0.81 & 30.00 \\
\hline 24 & Mu.Agaram & 0.80 & 28.00 \\
\hline 25 & Vetakudi & 1.20 & 29.00 \\
\hline 26 & Thottikuppam & 1.35 & 26.00 \\
\hline 27 & Siruvambar & 1.45 & 27.00 \\
\hline 28 & Thoravalur & 1.73 & 24.00 \\
\hline 29 & Sembalakurichi & 1.62 & 20.00 \\
\hline 30 & Puthukooraipetai & 1.45 & 46.00 \\
\hline \multirow[t]{4}{*}{31} & Rupanarayananallur & 1.40 & 45.00 \\
\hline & Range & $0.34-1.73$ & $24.00-55.00$ \\
\hline & Mean & 0.74 & 39.00 \\
\hline & Standerd Deviation & 0.33 & 9.00 \\
\hline
\end{tabular}


Table.3 Physical Quality Indicators of ICAR-KVK, Viruthachalam

\begin{tabular}{|c|c|c|c|c|c|c|c|c|c|c|}
\hline $\begin{array}{l}\text { Management } \\
\text { strategies }\end{array}$ & $\begin{array}{c}\text { Bulk } \\
\text { density }\end{array}$ & $\begin{array}{c}\text { Particle } \\
\text { density }\end{array}$ & porosity & $\begin{array}{c}\text { Sand } \\
\%\end{array}$ & $\begin{array}{c}\text { Silt } \\
\%\end{array}$ & $\begin{array}{c}\text { Clay } \\
\%\end{array}$ & $\begin{array}{c}\text { Soil } \\
\text { Texture }\end{array}$ & $\begin{array}{l}\text { Aggregate } \\
\text { stability }\end{array}$ & $\begin{array}{c}\text { Mean weight } \\
\text { diameter }\end{array}$ & WHC \\
\hline SRI Method & 1.33 & 2.76 & 45.20 & 53.0 & 18.0 & 47.0 & $\mathrm{cl}$ & 45.90 & 1.74 & 51.00 \\
\hline Organic Farming & 1.22 & 3.24 & 48.53 & 44.0 & 19.0 & 57.0 & $\mathrm{cl}$ & 48.50 & 1.83 & 58.00 \\
\hline Aerobic Rice & 1.25 & 3.25 & 40.00 & 36.0 & 24.0 & 43.0 & $\mathrm{cl}$ & 47.20 & 1.72 & 52.00 \\
\hline $\begin{array}{l}\text { Conventional } \\
\text { Farming }\end{array}$ & 1.23 & 3.21 & 38.30 & 24.0 & 23.0 & 31.0 & $\mathrm{scl}$ & 42.30 & 1.70 & 45.00 \\
\hline RANGE & $\begin{array}{l}1.21- \\
1.33\end{array}$ & $2.76-3.25$ & $\begin{array}{c}38.00- \\
48.53\end{array}$ & $\begin{array}{l}53.0- \\
24.0\end{array}$ & $\begin{array}{l}14.0- \\
24.0\end{array}$ & $\begin{array}{l}57.0- \\
30.0\end{array}$ & & $42.30-49.40$ & $1.69-1.85$ & $45-58$ \\
\hline MEAN & 1.24 & 3.15 & 41.57 & 37.0 & 20.0 & 43.0 & $\begin{array}{l}\text { Clay } \\
\text { loam }\end{array}$ & 46.00 & 1.75 & 51.00 \\
\hline STD. DEVIATION & 0.04 & 0.19 & 4.29 & 9.0 & 3.0 & 11.0 & & 2.64 & 0.06 & 4.84 \\
\hline
\end{tabular}


Table.4 Principal components, Eigen values and component matrix variables of Cuddalore district

\begin{tabular}{|l|c|c|c|c|c|}
\hline Principal components & PC 1 & PC 2 & PC 3 & PC 4 & PC 5 \\
\hline Eigen values & 13.166 & 52.663 & 52.663 & 3.748 & 14.991 \\
\hline \%Variance & 67.654 & 2.149 & 8.596 & 76.25 & 1.071 \\
\hline \%Cumulative & 4.284 & 80.534 & 0.899 & 3.597 & 84.131 \\
\hline variance & & & & & \\
\hline Weightage factor & 0.833 & 0.705 & 0.54 & 0.422 & 0.303 \\
\hline Bulk Density & -0.095 & $\mathbf{- 0 . 3 4 2}$ & -0.072 & 0.169 & $\mathbf{0 . 5 3 8}$ \\
\hline Particle Density & -0.145 & 0.079 & $\mathbf{- 0 . 4 8 6}$ & -0.117 & 0.173 \\
\hline Porosity & -0.035 & 0.301 & $\mathbf{- 0 . 3 8}$ & $-\mathbf{0 . 3 9 5}$ & -0.287 \\
\hline Sand & -0.075 & -0.152 & -0.248 & $\mathbf{0 . 4 4 1}$ & -0.188 \\
\hline Silt & -0.181 & 0.282 & 0.193 & -0.152 & 0.087 \\
\hline Clay & 0.11 & -0.266 & 0.241 & -0.424 & -0.285 \\
\hline AWC & -0.026 & $\mathbf{- 0 . 3 5 3}$ & -0.273 & -0.372 & -0.08 \\
\hline MWD & $\mathbf{- . 2 6 4}$ & 0.052 & 0.093 & 0.071 & 0.059 \\
\hline Aggregate stability & -0.252 & 0.075 & 0.015 & 0.065 & -0.097 \\
\hline P & -0.168 & -0.182 & 0.341 & 0.054 & -0.278 \\
\hline EC & -0.205 & 0.17 & 0.168 & 0.081 & 0.098 \\
\hline OC & -0.2 & -0.293 & 0.057 & -0.005 & -0.132 \\
\hline CEC & -0.2 & -0.273 & -0.048 & -0.026 & -0.016 \\
\hline AN & -0.231 & -0.15 & 0.243 & -0.109 & -0.055 \\
\hline AP & -0.136 & -0.074 & 0.221 & $-\mathbf{0 . 4 1 5}$ & $\mathbf{0 . 4 7 2}$ \\
\hline AK & -0.255 & 0.142 & -0.035 & -0.028 & -0.026 \\
\hline TN & -0.188 & -0.259 & -0.256 & -0.029 & 0.122 \\
\hline Zn & $\mathbf{- 0 . 2 6 5}$ & 0.117 & -0.018 & 0.017 & 0.007 \\
\hline Fe & $-\mathbf{0 . 2 6 5}$ & 0.03 & -0.114 & -0.051 & -0.051 \\
\hline Boron & -0.251 & 0.184 & -0.052 & 0.023 & 0.03 \\
\hline MBC & -0.171 & 0.179 & 0.033 & -0.171 & 0.02 \\
\hline MBN & -0.209 & -0.199 & -0.156 & -0.007 & -0.191 \\
\hline PMN & -0.26 & 0.136 & 0.062 & 0.077 & -0.024 \\
\hline SRR & -0.251 & -0.05 & 0.007 & -0.031 & 0.033 \\
\hline DHA & -0.236 & -0.053 & 0.062 & 0.142 & -0.257 \\
\hline & & & & & \\
\hline
\end{tabular}

Table.5 Cuddalore District highly weighed parameters under Principal component Analysis

\begin{tabular}{|c|c|c|c|c|c|}
\hline $\begin{array}{c}\text { Highy weighed } \\
\text { parameters }\end{array}$ & PC 1 & PC 2 & PC 3 & PC 4 & PC 5 \\
\hline & $\begin{array}{l}\text { Bulk } \\
\text { density }\end{array}$ & $\begin{array}{l}\text { Particle } \\
\text { density }\end{array}$ & Porosity & WHC & Sand \\
\hline
\end{tabular}


Fig.1 Cuddalore district of Tamil Nadu

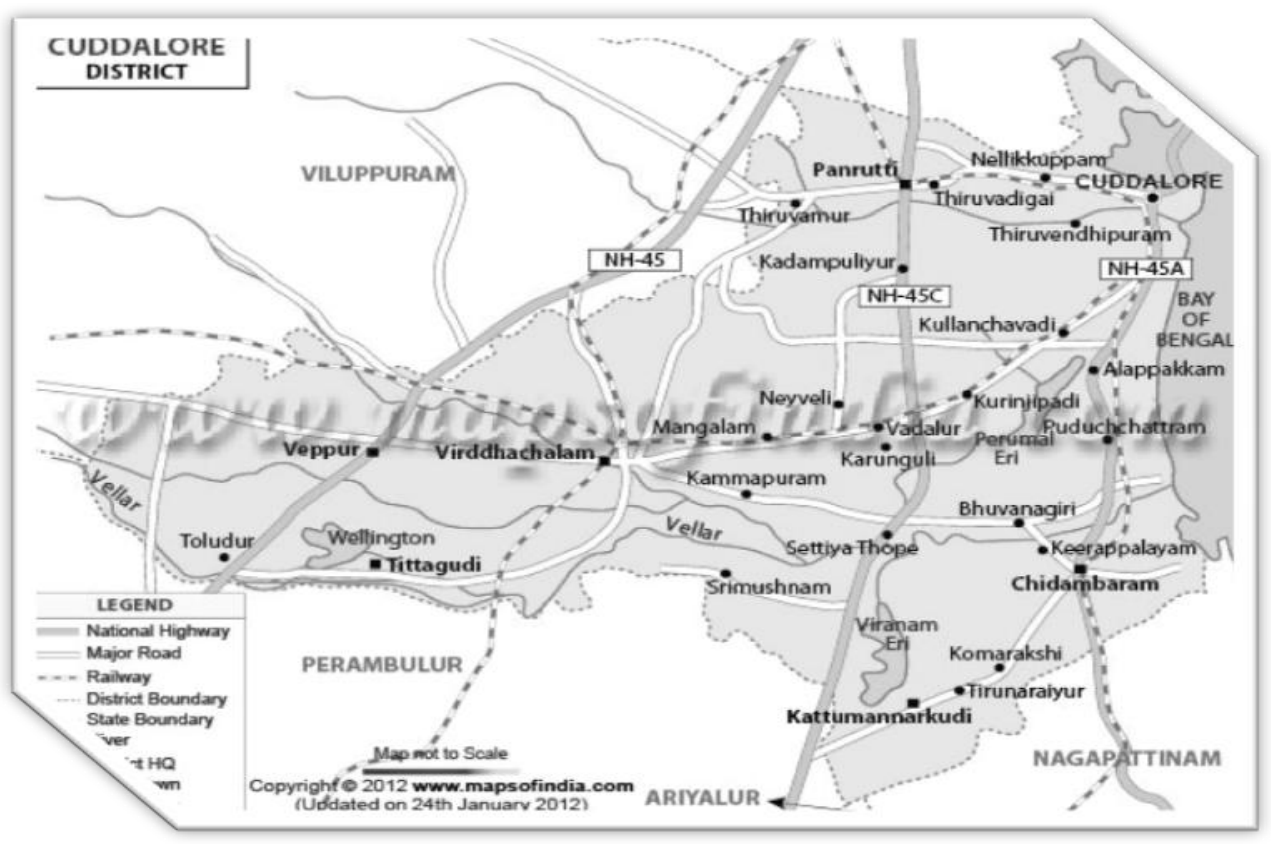

Fig.2

PCA graph of variables

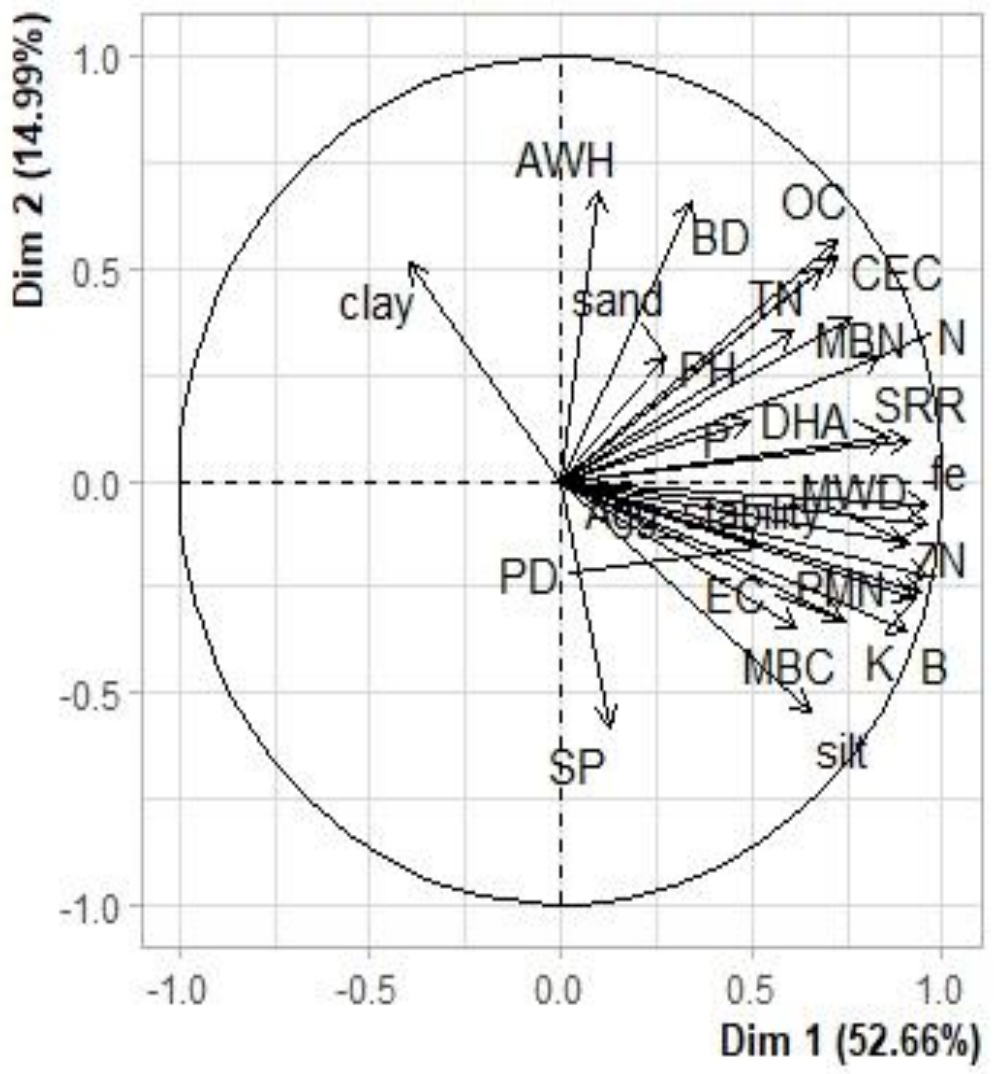


Fig.3

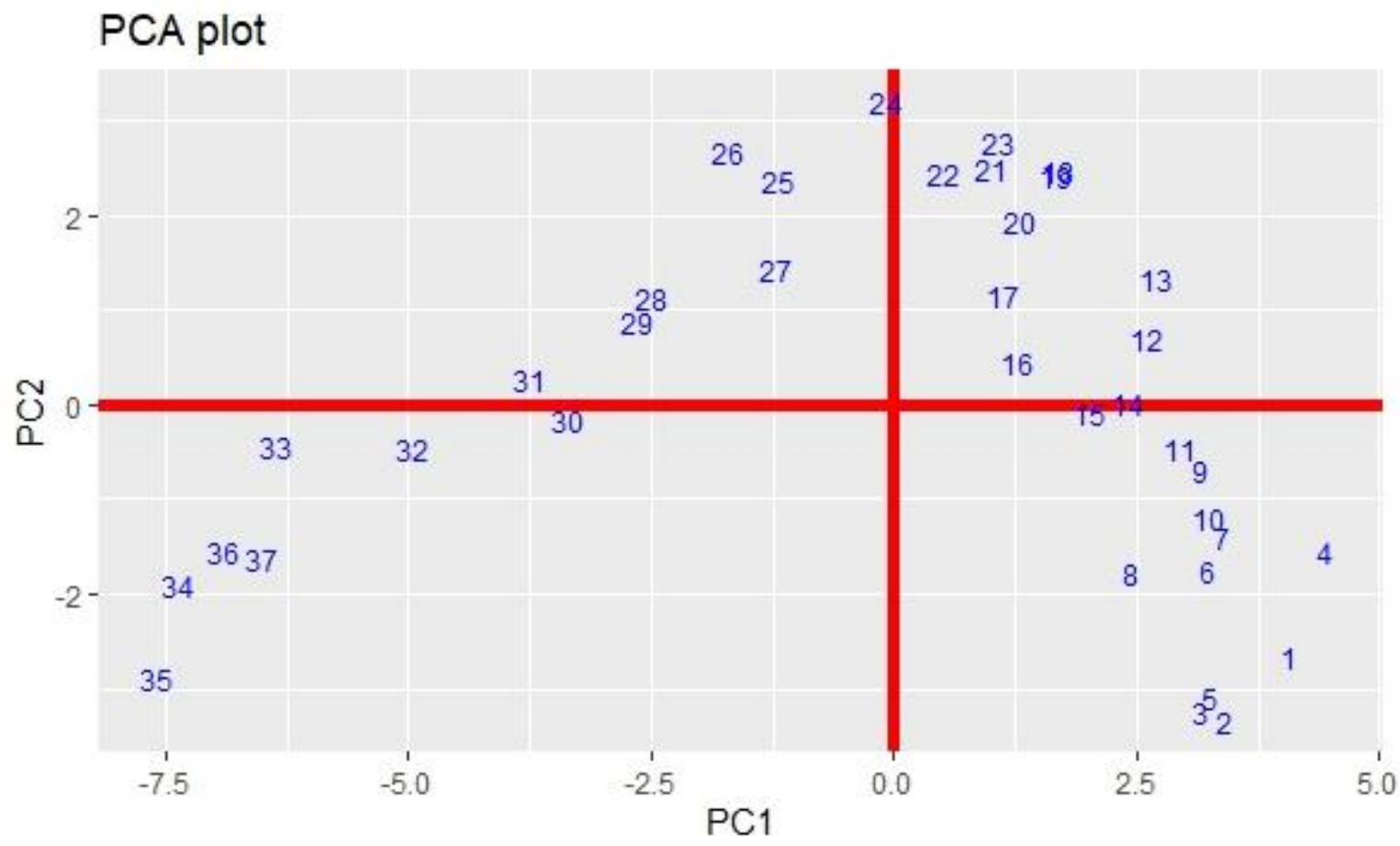

Water holding capacity (WHC) varied from 24.00 to 55.00 per cent. Under organic farming practice of rice cultivation 58.00 per cent was registered the highest water holding capacity.

\section{Principal component analysis}

The results obtained from PCA indicated five Principal Components ( PCs) with eigen values greater than 1 (Table 4) and soil variables from each PC were considered for minimum soil data set MDS) The soil parameters selected from PC 1, PC2, PC3, PC4, PC5 were, bulk density, particle density, porosity, WHC, sand, Aggregate stability, Mean Weight Diameter

However, PCA Pt, PCA graph variables showed higher variables between these parameters indicated available phosphorus which has the highest factor loading was retained in the MDS.
In conclusion, soil quality index is a useful tool to assess soil health and well being. Few methods are available to estimate it. Among those PCA based scoring, ranking and weightage method gaining popularity. However, SQI assessment primarily depends on objectives of study or soil functions need to be addressed. Selection of MDS and its ranking play important role for determining SQI. Cuddalore district soil Physical quality indicators soil bulk density high under based on the Principal Component Analysis.

\section{References}

Andrews, S.S., Karlen, D.L. and Cambardella, C.A. 2004. The soil management assessment framework: A quantitative evaluation using case studies. Soil Science Society of America Journal. 68, 1945-1962.

Andrews, S.S., Karlen, D.L., Mitchell, J.P., 2002. A comparison of soil quality indexing methods for vegetable 
production systems in northern California. Agric. Ecosyst. Environ. 90, 25-45.

Arshad, M.A. and Martin, S. 2002. Identifying critical limits for soil quality indicators in agro-ecosystems. Agriculture, Ecosystems and Environment. 88, 153-1.

Bandyopadhyay, P. K., Saha, Subita and Mallick, S. (2011). Comparison of Soil Physical Properties between a Permanent Fallow and a Long-Term Rice-Wheat Cropping with Inorganic and Organic Inputs in the Humid Subtropics of Eastern India', Communications in Soil Science and Plant Analysis, 42: 4, 435 - 449.

Chaudhury, J., Mandal, U.K., Sharma, K. L., Ghosh, H. and Mandal, B. 2005. Assessing Soil Quality Under LongTerm Rice Based Cropping System. Communications in Soil Science and Plant Analysis. 36, 1141-1161.

Doran, J. W. and Parkin. T. B. 1996. Quantitative indicators of soil quality: A minimum data set. In: Methods for Assessing Soil Quality, (J. W. Doran and A. J. Jones, Eds.). Soil Science Society of America, Special Publication No. 49, Madison, Wisconsin, USA, pp. 25-37.

Doran, J.W. 2002. Soil health and global sustainability: translating science into practice. Agriculture, Ecosystems and Environment. 88, 119-127.

Doran, J.W. and Parkin, T.B. 1994. Defining and assessing soil quality. In: Defining Soil Quality for a Sustainable Environment. Doran, J.W., Coleman, D.C., Bezdicek, D.F. and Stewart, B.A. (eds.), SSSA Special Publication No. 35, ASA and SSSA, Madison, WI. 3-21 pp.

Jackson M L. 1973. Soil chemical analysis. Prentice hall of India Pvt Ltd., New Delhi.

Karlen, D.L., Parkin T.P. and Eash, N.S. 1996. Use of soil quality indicators to evaluate conservation reserve program sites in Iowa. In: Doran, J.W., Jones, A.J. (Eds.), Methods for Assessing Soil Quality. SSSA Special Publication No. 49. SSSA, Madison, WI, pp. 345-355.

\section{How to cite this article:}

Seevagan, L., R.K. Kaleeswari, M.R. Backiyavathy and Balachandar, D. 2020. Assessment of Soil Physical Quality Indicators in Rice Soils of Cuddalore District of Tamil Nadu, India. Int.J.Curr.Microbiol.App.Sci. 9(07): 1476-1485. doi: https://doi.org/10.20546/ijcmas.2020.907.169 